\title{
Classificação Internacional de Funcionalidade, Incapacidade e Saúde em Unidade de Terapia Intensiva: Proposta de avaliação fisioterapêutica
}

\author{
International Classification of Functioning, Disability, and Health in the \\ Intensive Care Unit: a proposal for physiotherapy assessment
}

Lucas dos Santos Galaverna ${ }^{1}$; Eliane Maria de Carvalho ${ }^{2}$; Lilian de Fátima Dornelas ${ }^{2}$

${ }^{1 .}$ Graduando em Fisioterapia pela Faculdade de Educação Física e Fisioterapia da Universidade Federal de Uberlândia, Minas Gerais (MG), Brasil.

2. Docente do curso de Fisioterapia pela Faculdade de Educação Física e Fisioterapia da Universidade Federal de Uberlândia, Minas Gerais (MG), Brasil.

\section{Resumo}

Introdução: O fisioterapeuta possui competências para avaliar pacientes em Unidade de Terapia Intensiva (UTI) e a Classificação Internacional de Funcionalidade, Incapacidade e Saúde (CIF) permite norteá-lo no contexto biopsicossocial do indivíduo. Objetivo: Criar uma ficha de avaliação fisioterapêutica para UTI baseada no modelo conceitual da CIF, inserindo as categorias dos componentes de saúde para classificação. Metodologia: Estudo descritivo, qualitativo, que propõe uma ficha de avaliação fisioterapêutica utilizando o modelo conceitual da CIF, realizado em 4 fases: busca referente a utilização da CIF no ambiente hospitalar; procura de protocolos já utilizados para avaliação fisioterapêutica em UTIs; seleção dos componentes de saúde para a ficha proposta; e realização do método de ligação com as categorias da CIF, com a estruturação lógica da ficha. Resultados: A proposta contou com 54 categorias da CIF, 9 de atividade e participação (d), 31 de função corporal (b), 7 de estrutura corporal (s) e 7 de fatores ambientais (e). Nela, há um diagrama o qual propõe que o profissional descreva em cada componente de saúde o que será avaliado, bem como seções de identificação do paciente, histórico da doença, exame físico, diagnóstico fisioterapêutico e objetivos. Conclusão: A ficha de Avaliação Fisioterapêutica para UTI, construída sob as perspectivas qualitativa e quantitativa da CIF, facilita sua implementação em ambiente hospitalar e melhora a comunicação entre os profissionais de saúde, além de permitir que o fisioterapeuta elabore o diagnóstico e trace objetivos e condutas centrado no indivíduo, independente da sua condição de saúde.

Palavras-Chave: Classificação Internacional de Funcionalidade; Incapacidade e saúde; Unidades de Terapia Intensiva; Modalidades de Fisioterapia.

Autor correspondente:

Lucas dos Santos Galaverna

Endereço: Rua Pedro Silotto, 108 - Centro

Recebido em: 18/08/2020

CEP:13930-000-Serra Negra (SP), Brasil.

Revisado em: 10/11/2020

Aceito em: 13/11/2020

E-mail: lucasgalaverna98@hotmail.com

Publicado em: 02/03/2021 


\begin{abstract}
Introduction: Physiotherapists have the skills to assess patients in the Intensive Care Unit (ICU) and the International Classification of Functioning, Disability, and Health (ICF) allows them to be guided by the individual's biopsychosocial context. Objectives: To create a physiotherapy assessment form for the ICU based on the ICF conceptual model, inserting the categories of health components for classification. Methodology: A descriptive, qualitative study, which proposes a physiotherapy assessment form using the ICF conceptual model, carried out in 4 phases: finding the current use of ICF in the hospital environment; searching for protocols already used for physiotherapy assessments in ICUs; selecting health components for the proposed form; and performing the connection method with the categories of the ICF, using the logical structure of the form. Results: The proposal included 54 categories of the ICF, with 9 of these being activity and participation $(d), 31$ related to body functioning (b), 7 to body structure (s), and 7 to environmental factors (e). In this proposal, there is a diagram which proposes that the professional describe in each health component what will be assessed, as well as sections of patient identification, history of the disease, physical examination, physiotherapeutic diagnosis, and objectives. Conclusion: The Physiotherapy Assessment Form for the ICU, built under the qualitative and quantitative perspectives of the ICF, can facilitate its implementation in a hospital environment and improve communication between health professionals, in addition to allowing the physiotherapist to elaborate the diagnosis and define individual-centered objectives and conducts, regardless of their health condition.
\end{abstract}

Keywords: International Classification of Functioning, Disability and Health; Intensive Care Units; Physical Therapy Modalities.

\section{Introdução}

Em Unidades de Terapia Intensiva (UTI), a equipe de profissionais de saúde deve manter vigilância integral e alto monitoramento dos pacientes internados, tendo como premissa minimizar a permanência prolongada no leito e aumentar a sobrevida ${ }^{1}$. Nessa equipe, o papel do fisioterapeuta consiste em prevenir e tratar alterações osteomioarticulares e respiratórias decorrentes do tempo de internação, além de integrar ações da equipe multiprofissional sob abordagem interdisciplinar ${ }^{2}$.

Assim, o fisioterapeuta tem como competência avaliar e identificar os problemas das estruturas e funções corporais, limitações das capacidades e restrições de desempenho decorrentes da condição de saúde e definir metas a partir da conclusão da avaliação ${ }^{3}$. Para padronizar e utilizar uma linguagem comum durante a avaliação fisioterapêutica, o Conselho Federal de Fisioterapia e Terapia Ocupacional (COFFITO) recomenda a utilização da Classificação Internacional de Funcionalidade, Incapacidade e Saúde (CIF) para nortear este processo. ${ }^{4} \mathrm{~A}$ CIF é um sistema de classificação para a funcionalidade e incapacidade humana criada pela Organização Mundial da Saúde (OMS) em 2001. Seu modelo conceitual fornece um olhar ampliado sob o indivíduo, além da condição de saúde $^{5}$. Os domínios de saúde que compõem esta perspectiva são as funções e estruturas corporais, atividade, participação e os fatores contextuais ${ }^{6}$, e o resultado desta interação considera o que o indivíduo é capaz ou não de realizar em seu dia a dia ${ }^{7}$.

A utilização da CIF no ambiente de UTI tem sido disseminada, mas poucos estudos abordam sua aplicabilidade em ambiente hospitalar ${ }^{8}$. Os pacientes internados em UTI apresentam diversas alterações estruturais e funcionais do corpo, as quais, apesar de diagnosticadas, não são avaliadas muitas vezes, com reprodutibilidade entre os profissionais de saúde, incluindo fisioterapeutas ${ }^{9}$. Utilizar a CIF na visão qualitativa, enquanto modelo conceitual e na perspectiva quantitativa quanto à utilização das categorias para classificação dos componentes de saúde é imprescindível para delinear o cuidado com pacientes críticos diante da heterogeneidade de acometimentos encontrados. 
Diante disso, o presente estudo teve por objetivo criar uma ficha de avaliação fisioterapêutica para UTI baseada no modelo conceitual da CIF, inserindo as categorias dos componentes de saúde para classificação e qualificação.

\section{Metodologia}

Estudo descritivo, qualitativo realizado por meio da construção de uma ficha de avaliação fisioterapêutica baseada no modelo conceitual da CIF, utilizando o método de ligação entre os instrumentos utilizados para avaliação fisioterapêutica na UTI, com os componentes do modelo biopsicossocial da CIF e suas respectivas categorias para a classificação. Foi utilizado como referencial teórico as orientações de Cieza et al ${ }^{10}$ que propõem oito regras para ligação entre as medidas de resultado (instrumentos de avaliação de desfechos, relatos de usuários, exames de imagem, etc.) com a CIF. As regras determinam que todas as medidas de resultado devem ser consideradas antes de se realizar a ligação com as categorias da CIF. A construção da ficha de avaliação fisioterapêutica se deu em 4 fases:

$\mathbf{1}^{a}$ fase: Discussão entre os autores sobre a utilização da CIF em ambiente de Terapia Intensiva, com aprofundamento teórico sobre sua aplicação como um método de avaliação da funcionalidade e incapacidade, bem como a busca ativa em base de dados que utilizaram esse instrumento neste ambiente e sua viabilidade de aplicação.

$\mathbf{2}^{\mathbf{a}}$ fase: Busca por protocolos de avaliação fisioterapêutica em UTI e aprofundamento teórico sobre os sistemas neurológico, cardiorrespiratório, hemodinâmico, ventilatório e osteomioarticular, úteis a serem avaliados neste contexto, além de dados sobre as atividades diárias e informações de fisioterapeutas com experiência clínica em ambiente hospitalar, para determinação de quais componentes seriam imprescindíveis para a avaliação.

$\mathbf{3}^{\mathbf{a}}$ fase: Seleção dos componentes de saúde adequados e relevantes para a avaliação fisioterapêutica em UTI com a inserção das categorias para a classificação.

$4^{\text {a }}$ fase: Estruturação da ficha de avaliação fisioterapêutica no programa Microsoft@ Word $@$ com articulação dos dados de avaliação e organização estrutural do novo instrumento para que a avaliação siga um raciocínio lógico e prático para a utilização.

Ao final, a ficha de avaliação foi discutida entre os autores, buscando maior praticidade e viabilidade da sua aplicação.

Em nenhum momento da etapa de construção da ficha de avaliação houve conflito de interesses entre os autores e suas instituições.

\section{Resultados}

A ficha de avaliação fisioterapêutica em UTI (Anexo 1) apresenta-se dividida em 6 partes, quais sejam: diagrama, identificação do paciente, anamnese, exame físico, diagnóstico fisioterapêutico e objetivos de tratamento. No total, foram incluídas 54 categorias da CIF, 9 de atividade e participação (d), 31 de função corporal (b), 7 de estrutura corporal (s) e 7 de fatores ambientais (e). (FIGURA 1) 


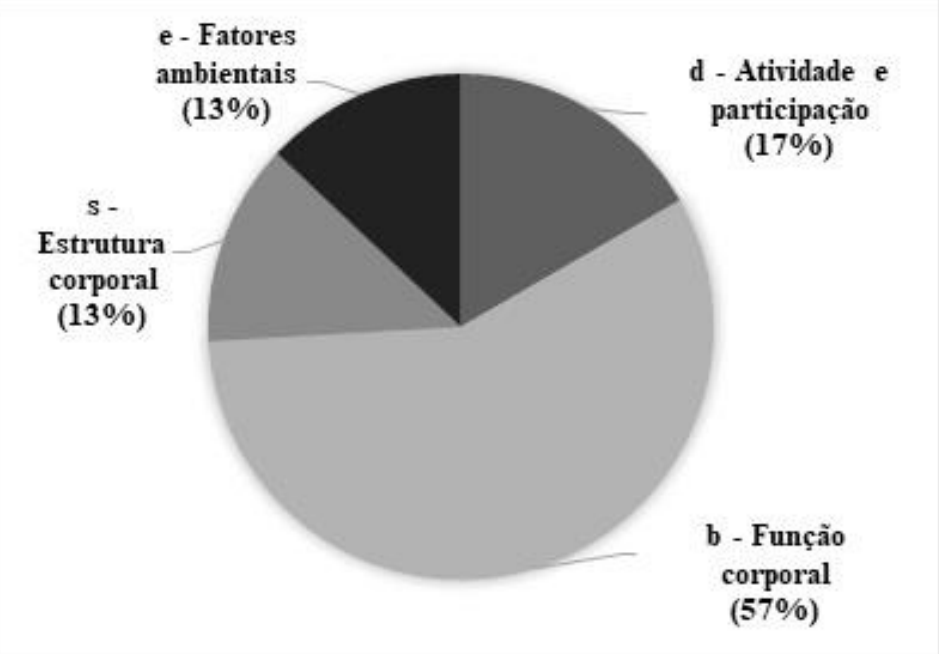

FIGURA 1: Porcentagem de categorias contempladas na ficha de avaliação fisioterapêutica em UTI

O diagrama inicial (FIGURA 2) propõe o que será avaliado em cada componente de saúde, especificando a condição de saúde baseada no Código Internacional de Doença (CID-10), os problemas em estruturas e funções corporais, as limitações de capacidade, as restrições de desempenho e os fatores ambientais - os quais podem se comportar como barreira ou facilitador. Os fatores pessoais são preenchidos na ficha na seção de identificação do paciente com nome, idade, raça, data de nascimento, sexo, profissão, estado civil, nome do acompanhante, naturalidade, religião, escolaridade, endereço e contato pessoal.

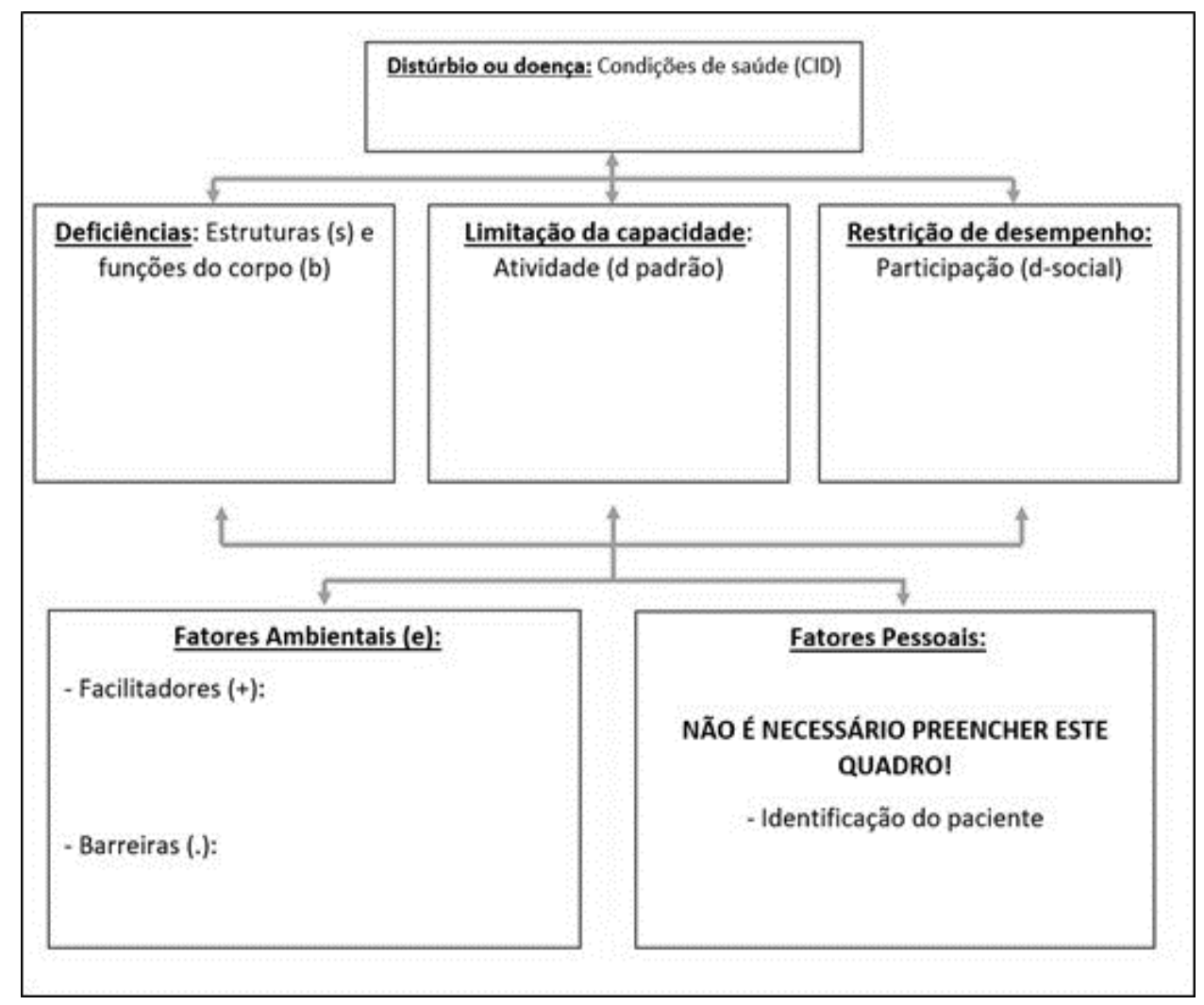

FIGURA 2: Diagrama conceitual da CIF que será preenchido para seleção das categorias que serão avaliadas 
A partir disso, o avaliador elege como avaliar por meio de instrumentos, questionários ou testes. O exame físico constituiu a maior parte da ficha de avaliação. Nele, foram incluídas categorias de todos os componentes classificáveis pela CIF (TABELA 1), incluindo função e estrutura corporais, atividade, participação e fatores ambientais. Ao final da ficha, o avaliador conta com um espaço para descrição do diagnóstico fisioterapêutico baseado na CIF.

TABELA 1: Categorias abordadas na ficha de avaliação fisioterapêutica para UTI

\begin{tabular}{|c|c|}
\hline \multicolumn{2}{|r|}{ s - Estrutura corporal } \\
\hline s198 & Estrutura do sistema nervoso, outra especificada \\
\hline s199 & Estrutura do sistema nervoso não especificada \\
\hline s430 & Estrutura do aparelho respiratório \\
\hline s540 & Estrutura dos intestinos \\
\hline s7608 & Estrutura do tronco, outra especificada \\
\hline s770 & Estrutura musculoesquelética relacionadas ao movimento \\
\hline s798 & Estrutura relacionada com o movimento, especificada \\
\hline \multicolumn{2}{|r|}{ b - Função corporal } \\
\hline b110 & Funções da consciência \\
\hline b1300 & Nível de energia \\
\hline b156 & Funções da percepção \\
\hline b265 & Função sensorial tátil \\
\hline b280 & Sensação de dor \\
\hline b310 & Funções da fala \\
\hline b410 & Funções cardíacas \\
\hline b4100 & Frequência cardíaca \\
\hline b4151 & Funções dos capilares \\
\hline b420 & Funções da pressão arterial \\
\hline b4301 & Funções de transporte de oxigênio pelo sangue \\
\hline b4352 & Funções dos vasos linfáticos \\
\hline b440 & Funções da respiração \\
\hline b4400 & Frequência respiratória \\
\hline b4402 & Profundidade da respiração \\
\hline b4452 & Funções dos músculos respiratórios acessórios \\
\hline b449 & Funções do ap. resp. especificadas e não especificadas \\
\hline b450 & Funções respiratórias adicionais \\
\hline b499 & Funções do aparelho cardiovascular, não especificada \\
\hline b5152 & Absorção de nutrientes \\
\hline b530 & Funções de manutenção do peso \\
\hline b5401 & Metabolismo dos hidratos de carbono \\
\hline b5403 & Metabolismo dos lipídeos \\
\hline b545 & Funções do equilíbrio hídrico, mineral e eletrolítico \\
\hline b610 & Funções de excreção urinária \\
\hline
\end{tabular}




$\begin{array}{ll}\text { b710 } & \text { Mobilidade das articulações } \\ \text { b730 } & \text { Funções da força muscular } \\ \text { b750 } & \text { Funções de reflexos motores } \\ \text { b760 } & \text { Funções do controle do movimento voluntário } \\ \text { b810 } & \text { Funções protetoras da pele } \\ \text { b820 } & \text { Funções reparadoras da pele }\end{array}$

d - Atividade e Participação

\begin{tabular}{ll}
\hline $\mathrm{d} 330$ & Falar \\
$\mathrm{d} 4103$ & Sentar-se \\
$\mathrm{d} 4104$ & Pôr-se em pé \\
$\mathrm{d} 4150$ & Permanecer deitado \\
$\mathrm{d} 420$ & Auto transferências \\
$\mathrm{d} 450$ & Andar \\
$\mathrm{d} 510$ & Lavar-se \\
$\mathrm{d} 550$ & Comer \\
$\mathrm{d} 820$ & Educação escolar \\
\hline
\end{tabular}

\begin{tabular}{ll}
\hline & \multicolumn{1}{c}{ e - Fatores ambientais } \\
\hline e110 & Produtos ou substancia para consumo pessoal \\
e1101 & Medicamentos \\
e198 & Produtos ou substancia para consumo pessoal, outro especificado \\
e199 & Produtos e tecnologias, outros especificados \\
e399 & Produtos e tecnologias, outros não especificados \\
e5800 & Apoio e relacionamentos, não especificados \\
\hline
\end{tabular}

\section{Discussão}

A criação da ficha de Avaliação Fisioterapêutica para UTI baseou-se no modelo conceitual e de classificação da CIF. Na perspectiva qualitativa, permite que os profissionais de saúde entendam as relações interacionais entre capacidade e desempenho relacionados aos fatores contextuais do indivíduo e não somente os aspectos biomédicos da condição de saúde ${ }^{11}$. Já na perspectiva quantitativa, busca-se implementar o uso das categorias dos componentes de saúde para qualificação, melhorando a comunicação entre os profissionais de saúde em uma linguagem padronizada ${ }^{12,13}$.
Para a elaboração da ficha, o componente função corporal corresponde $57 \%$ de toda a avaliação, comtemplado por meio de instrumentos amplamente disseminados em UTI, como as escalas de Rass, de Ramsey, escala Perme de mobilidade e escala de coma de Glasgow $^{14}$ 15, 16, 17. Ainda se observa a tendência de abordar a avaliação em UTI baseada em componentes centrados no modelo biomédico ${ }^{11,17,18,19,20}$. No entanto, a presente ficha de avaliação apresenta um panorama ampliado no qual contém todas categorias pertinentes ao ambiente hospitalar e propõe ao profissional elencar apenas os componentes necessários para uma abordagem centrada no indivíduo e não na condição de saúde. ${ }^{17,21}$ 
A estruturação de uma avaliação que mensure aspectos biopsicossociais condizentes com a realidade da UTI possibilita o delineamento das necessidades dos pacientes sob uma abordagem humanizada, com resolutividade e sob o enfoque da integralidade do cuidado $^{22}$. Neste contexto, $17 \%$ da ficha contempla os domínios atividade e participação e são os que mais dialogam no âmbito da Fisioterapia ${ }^{6,23}$. As categorias referentes a atividades de autocuidado podem indicar perdas na independência que impactam na qualidade de vida como o banhar-se (d510) e alimentar-se (d550). Além disso, categorias como a fala (d330), o andar (d450), pôrse em pé (d4104), sentar (d41003) e capacidade de realizar auto transferências (d420) permitem nortear o profissional sobre as reais necessidades do paciente no que ele é capaz de fazer ou não no seu dia a $\operatorname{dia}^{20}$. Embora as circunstancias e a rotina dos pacientes internados em UTIs podem dificultar a avaliação dos domínios de atividade e participação, pois a maioria dos indivíduos se encontram em estado comatoso, com uso de bloqueadores neuromusculares, dores ou em condições clínicas que impedem a comunicação e autonomia ${ }^{24}$, é preciso observar e questionar os acompanhantes, familiares e os outros profissionais que lidam com o paciente, pois estas informações são determinantes para a mensuração da eficácia terapêutica e delimitação do prognóstico ${ }^{25}$.

Quanto aos fatores ambientais, $17 \%$ da ficha de avaliação apresenta categorias referentes ao contexto de vida que podem se comportar como facilitadores ou barreiras, tais como, ter ou não o acesso aos serviços de saúde (e5800), apoio familiar (e399) e uso de medicamentos (e1101). Essas categorias podem representar sua importância no momento da discussão de condutas e necessidade de encaminhamentos, e por mais que o fisioterapeuta de UTI tende a não considerar estas questões extra hospitalares no seu processo avaliativo, incluí-las na ficha de avaliação tem o propósito de ampliar a visão do profissional, desmistificando a rotina mecanicista e abordagem fragmentada que é muito vista no ambiente hospitalar ${ }^{26}$.

Contudo, o processo de implementação da CIF no ambiente hospitalar demanda conhecimento e entendimento dos profissionais de saúde em utilizá-la tanto no âmbito conceitual quanto no uso dos qualificadores ${ }^{12,13,22}$. Nesse contexto, a proposta da ficha de avaliação fisioterapêutica para UTI vem facilitar seu uso, pois o seu formato permite utilizar tanto a perspectiva qualitativa quanto quantitativa. A intenção ao construir a ficha baseada na CIF é disseminar o seu uso mostrando o quão é relevante avaliar o indivíduo além da sua condição de saúde, que ao usá-la, não demandará tempo extra para qualificar as categorias e para padronizar a linguagem da equipe.

\section{Conclusão}

A ficha de Avaliação Fisioterapêutica para UTI, construída sob as perspectivas qualitativa e quantitativa da CIF, facilita a implementação dessa classificação em ambiente hospitalar e melhora a comunicação entre os profissionais de saúde, além de permitir que o fisioterapeuta elabore o diagnóstico e trace objetivos e condutas centrados no indivíduo, independentemente da sua condição de saúde.

\section{Referências}

1- FRANÇA, E. E. T. FERRARI, F.; FERNANDES, P.; CAVALCANTI, R.; DUARTE, A.; MARTINEZ, B. P. et al. Physical therapy in critically ill adult patients: recommendations from the Brazilian Association of Intensive Care Medicine Department of Physical Therapy. Revista

Brasileira de Terapia Intensiva, v. 24, n. 1, p. 6-22, 2012. Disponível em: <https://www.scielo.br/pdf/rbti/v24n1/en_03.pd f> Acesso em: 14 jun. 2020.

\section{2- MENEZES, S. Intensive Care Physical}

Therapy: a new name for an old specialty. ASSOBRAFIR Ciência, v. 2, n. 2, p. 49-53, 2011. Disponível em: <http://www.uel.br/revistas/uel/index.php/rebraf 
is/article/download/10602/9308> Acesso em: 14 jun. 2020 .

3- SAMPAIO, R. F.; MANCINI, M. C.; FONSECA, S. T. Produção científica e atuação profissional: aspectos que limitam essa integração na fisioterapia e na terapia ocupacional. Revista Brasileira de

Fisioterapia, v. 6 n. 3, p. 113-8, 2012.

Disponível em:

<http://www.revistas.usp.br/fpusp/article/downl $\underline{\mathrm{oad} / 78113 / 82201 /}$

especializacaofisioto/files/2010/03/Producaocie ntifica-e-atuacao-profissional-RBF-20021.pdf>

Acesso em 14 jun. 2020.

4- BERNARDES, J. M.; PEREIRA, A. A. The International Classification of Functioning, Disability and Health (ICF) and its contributions to physical therapy. Fisioterapia Brasil, v. 12 n. 1, p. 58-64, 2011. Disponível em:

<https://portalatlanticaeditora.com.br/index.php/ fisioterapiabrasil/article/view/602 > Acesso em: 14 jun. 2020.

5- Organização Mundial de Saúde. Rumo a uma linguagem comum para funcionalidade, incapacidade e saúde: CIF. Genebra: OMS/WHO, 2002.

6- Organização Mundial de Saúde (OMS). Organização Panamericana de Saúde (OPAS). CIF classificação internacional de funcionalidade, incapacidade e saúde. Universidade de São Paulo. São Paulo: Edusp, 2008.

7- BROCKOW, T.; CIEZA, A.; KUHLOW, H.; SIGL, T.; FRANKE, T.; HARDER, M. et al. Identifying the concepts contained in outcome measures of clinical trials on musculoskeletal disorders and chronic widespread pain using the international classification of functioning, disability and health as a reference. Journal Rehabilitation Medicine, v. 44, p. 30-36, 2004. Disponível em: <https://pubmed.ncbi.nlm.nih.gov/15370745/> Acesso em: 16 jun. 2020

8- CASTANEDA. L.; CASTRO, S. S. Brazilian publications on the International Classification of Functioning. Acta Fisiátrica, v. 20, n. 1 p. 29-36, 2013. Disponível em: $<$ https://www.actafisiatrica.org.br/detalhe_artig o.asp?id=492> Acesso em: 16 jun. 2020.

9- SILVA, B. N. O.; SOUZA, R. C.; CARVALHO, T. P. V.; PODEROSO NETO, M. L.; MACIEL, L. Y.S.; ESPINHEIRA, P. F. S. et al. Application of the international classification of functioning, disability and health in Cardiothoracic Intensive Care Unit. Fisioterapia Brasil, v. 17, n. 2, p. 107-118, 2016. Disponível em:

<https://portalatlanticaeditora.com.br/index.php/

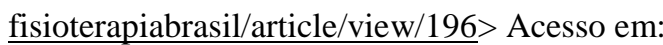
16 jun. 2020.

10- CIEZA, A.; BROCKOW, T.; EWERT, T.; AMMAN, E.; KOLLERITS, B.; CHATTERJI, S. et al. Linking health-status measurements to the internationalclassification of functioning, disability and health. Journal Rehabilitation Medicine, v. 34, p. 205-210, 2002. Disponível em: 〈https://core.ac.uk/reader/207503566> Acesso em: 25 jun 2020.

11- BIZ, M. C. P.; CHUN, R. Y. S.

Operationalization of the International Classification of Functioning, Disability and Health, ICF, in a Specialized Rehabilitation Center. CoDAS, v. 32, n. 2, 2020. Disponível em: 
$<$ https://www.scielo.br/scielo.php?pid=S2317$17822020000200301 \&$ script=sci_arttext>

Acesso em: 25 jun. 2020.

12- TEMPEST, S.; HARRIES, P.; KILBRIDE, C.; SOUZA, L. To adopt is to adapt: the process of implementing the ICF with an acute stroke multidisciplinary team in England. Disabilitie and Rehabilitation, v. 34, n. 20, p. 1686-94,

2012. Disponível em:

<https://pubmed.ncbi.nlm.nih.gov/22372376/>

Acesso em 27 jun. 2020.

13- STUCKI, G.; ZAMPOLINI, M.;

JUOCEVICIUS, A.; NEGRINI, S.;

CHRISTODOULOU, N. Practice, science and governance in interaction: European effort for the systemwide implementation of the International Classification of Functioning, Disability and Health (ICF) in Physical and Rehabilitation Medicine. European Journal of Physical and Rehabilitation Medicine, v. 53, n. 2, p. 299-307, 2017. Disponível em: <https://pubmed.ncbi.nlm.nih.gov/27882907/> Acesso em: 27 jun. 2020

14- NASSAR JUNIOR, A. P.; PIRES NETO, R. C.; FIGUEIREDO, W. B.; PARK, M. Validity, reliability and applicability of Portuguese versions of sedationagitation scales among critically ill patients. Sao Paulo Medical Journal, v. 126, n. 4, p. 215-9, 2008.

Disponível em:

<https://www.scielo.br/scielo.php?script=sci_art text\&pid=S1516-31802008000400003 > Acesso em: 27 jun. 2020.

15- CURZEL, J.; FORGIARINI JUNIOR, L. A.; RIEDER, M. M. Evaluation of functional independence after discharge from the intensive care unit. Revista Brasileira de Terapia Intensiva, v. 25, n. 2, p. 93-98, 2013.
Disponível em:

<https://www.scielo.br/scielo.php?pid=S0103507X2013000200006\&script=sci_abstract\&tlng $=\mathrm{pt}>$ Acesso em: 30 jun. 2020

16- CORDEIRO, A. L. L.; BRITO, A. A. O. R.; SANTANA, N. M. A.; SILVA, I. N. M. S.; NOGUEIRA, S. C. O.; GUIMARÃES, A. R. F. et al. Análise do grau de independência funcional pré e na alta da uti em pacientes submetidos à cirurgia cardíaca. Revista Pesquisa em Fisioterapia v. 5, n. 1, p. 21-27, 2015. Disponível em:

$<$ https://www5.bahiana.edu.br/index.php/fisiote rapia/article/view/574> Acesso em: 05 jul. 2020.

17- MATURANA, M. J.; ANTUNES, A. L.; BENTO, B. T. S.; RIBAS, P. R. S.; AQUIM, E. E. Scales of Functional Evaluation in Intensive Care Unit (ICU): Systematic Review. Revista Inspirar, v. 13, n. 2, p. 21-29, 2017. Disponível em:

<https://www.inspirar.com.br/revista/escalas$\underline{\text { de-avaliacao-funcional-em-unidade-de-terapia- }}$ intensiva-uti-revisao-sistematica/> Acesso em: 05 jul. 2020.

18- ALVES, G. A.; MARTINEZ, B. P.; LUNARDI, A. C. Assessment of the measurement properties of the Brazilian versions of the Functional Status Score for the ICU and the Functional Independence Measure in critically ill patients in the intensive care unit. Revista Brasileira de Terapia Intensiva. [Internet]. 2019 [cited 2020 jul 06]; v. 31, n. 4, p. 521-528, 2019. Disponível em: <https://www.scielo.br/scielo.php?pid=S0103$\underline{507 X 2019000400521 \& \text { script }=\text { sci } \text { arttext } \& \text { tlng }=}$ pt> Acesso em: 06 jul. 2020. 
19- O’SULLIVAN, S. B.; SCHMITZ, T. J.

Fisioterapia Avaliação e Tratamento. 4a edição. São Paulo: Manole, 2004.

20- SILVA, B. N. O.; SOUZA, R. C.; CARVALHO, T. P. V.; PODEROSO NETO. M. L.; MACIEL, L. Y. S.; ESPINHEIRA, P. F. S. et al. Application of the international classification of functioning, disability and health in Cardiothoracic Intensive Care Unit. Fisioterapia Brasil, v. 17, n. 2, p. 107-118, 2016. Disponível em: <https://portalatlanticaeditora.com.br/index.php/ fisioterapiabrasil/article/view/196> Acesso em: 16 jun. 2020

21- CHATTERJI, S.; BYLES, J.; CUTLER, D.; SEEMAN, T.; VERDES, E. Health, functioning, and disability in older adults present status and future implications. The Lancet, v. 385, n. 9967, p. 563-75, 2015.

Disponível em:

<http://dx.doi.org/10.1016/S0140-

6736(14)61462-8. PMid:25468158> Acesso em: 13 jul. 2020.

22- RUARO, J. A.; RUARO, M. B.; GUERRA, O. International Classification of Functioning, Disability and Health core set for physical health of older adults. Journal of Geriatric Physical Therapy, v. 37, n. 4, p. 147-53, 2014. Disponível em: <https://pubmed.ncbi.nlm.nih.gov/24495869/> Acesso em 13 jul. 2020.

23- TOLDRÁ, R. C. Reflexões acerca da Terapia Ocupacional na Atenção à Pessoa Portadora de Deficiência Física. Terapia Ocupacional: teoria e prática. Campinas: Papirus, 2003.

24- AUDISIO, R. A.; PAPA, D.; RAMESH, H. S.

J.; GENNARI, R.; LEEUWEN, B. L.;

CORSINI, G. et al. Shall we operate?
Preoperative assessment in elderly cancer patients (PACE) can help: a SIOG surgical task force prospective study. Critical Reviews Oncology/Hematology, v. 65, n. 2, p. 156-63, 2008. Disponível em: <https://pubmed.ncbi.nlm.nih.gov/18082416/> Acesso em: 13 jul. 2020.

25- FUKATA, S.; ANDO, M.; AMEMIYA, T.; KUROIWA, K.; ODA, K. Postoperative function following radical surgery in gastric and colorectal cancer patients over 80 years of age: an objection to "ageism". Nagoya Journal Medicine Science, v. 74, n. 3-4, p. 241-51, 2012. Disponível em:

<https://www.ncbi.nlm.nih.gov/pmc/articles/P MC4831233/> Acesso em: 14 jul. 2020.

26- SILVA, M. A. M.; PINHEIRO, A. K. B.; SOUZA, A. M. A.; MOREIRA, A. C. A. Health promotion in hospital settings. Revista

Brasileira de Enfermagem, v. 64, n. 3, p. 5969, 2011. Disponível em: <https://www.scielo.br/pdf/reben/v64n3/v64n3a 27.pdf> Acesso em 18 jul. 2020 